\title{
ARTICLE
}

\section{Predictors of depressive symptoms in persons with diabetic peripheral neuropathy: a longitudinal study}

\author{
L. Vileikyte • M. Peyrot • J. S. Gonzalez • R. R. Rubin • \\ A. P. Garrow $•$ D. Stickings $\cdot$ C. Waterman • \\ J. S. Ulbrecht • P. R. Cavanagh • A. J. M. Boulton
}

Received: 7 December 2008 / Accepted: 16 March 2009/Published online: 18 April 2009

(C) Springer-Verlag 2009

\begin{abstract}
Aims/hypothesis The aim of the study was to determine whether diabetic peripheral neuropathy (DPN) is a risk factor for depressive symptoms and examine the potential mechanisms for this relationship.

Methods This longitudinal study (9 and 18 month followup) of 338 DPN patients (mean age 61 years; $71 \%$ male; $73 \%$ type 2 diabetes) examined the temporal relationships between DPN severity (mean \pm SD; neuropathy disability score [NDS], 7.4 \pm 2.2 ; mean vibration perception threshold, $41.5 \pm 9.5 \mathrm{~V}$ ), DPN somatic experiences (symptoms and foot ulceration), DPN psychosocial consequences (restrictions in activities of daily living [ADL] and social selfperception) and the Hospital Anxiety and Depression subscale measuring depressive symptoms (HADS-D; mean 4.9 93.7$)$.
\end{abstract}

L. Vileikyte $(\bowtie) \cdot$ A. P. Garrow $\cdot$ D. Stickings $\cdot$ C. Waterman $\cdot$

A. J. M. Boulton

Department of Diabetes, University of Manchester,

193 Hathersage Road,

Manchester M13 0JE, UK

e-mail: lvileikyte@med.miami.edu

L. Vileikyte $\cdot$ A. J. M. Boulton

Division of Endocrinology, Diabetes and Metabolism,

University of Miami,

Miami, FL, USA

M. Peyrot

Department of Sociology, Loyola College,

Baltimore, MD, USA

M. Peyrot • R. R. Rubin

Departments of Medicine and Pediatrics,

Johns Hopkins University,

Baltimore, MD, USA
Results Controlling for baseline HADS-D and demographic/disease variables, NDS at baseline significantly predicted increased HADS-D over 18 months. This association was mediated by baseline unsteadiness, which was significantly associated with increased HADS-D. Baseline ADL restrictions significantly predicted increased HADS-D and partly mediated the association between baseline unsteadiness and change in HADS-D. Increased pain, unsteadiness and ADL restrictions from baseline to 9 months each significantly predicted increased HADS-D over 18 months. Change in social self-perception from baseline to 9 months significantly predicted increased HADS-D and partly mediated the relationships of change in unsteadiness and ADL restrictions with change in HADS-D.

Conclusions/interpretation These results confirm that neuropathy is a risk factor for depressive symptoms because it

\author{
J. S. Gonzalez \\ Departments of Psychology and Endocrinology, \\ Ferkauf Graduate School of Psychology and Albert Einstein \\ College of Medicine, \\ Yeshiva University, \\ New York, NY, USA
}

J. S. Ulbrecht

Department of Medicine and Biobehavioral Health, Pennsylvania State University,

University Park, PA, USA

P. R. Cavanagh

Department of Sports Medicine,

University of Washington,

Seattle, WA, USA 
generates pain and unsteadiness. Unsteadiness is the symptom with the strongest association with depression, and is linked to depressive symptoms by perceptions of diminished self-worth as a result of inability to perform social roles.

Keywords Activities of daily living · Depressive symptoms · Diabetic peripheral neuropathy $\cdot$ Longitudinal .

Social self-perception

$\begin{array}{ll}\text { Abbreviations } \\ \text { ADL } & \text { Activities of daily living } \\ \text { DPN } & \text { Diabetic Peripheral Neuropathy } \\ \text { HADS } & \text { Hospital Anxiety and Depression Scale } \\ \text { NDS } & \text { Neuropathy disability score } \\ \text { NeuroQoL } & \text { Neuropathy and Foot Ulcer-specific Quality } \\ & \text { of Life Instrument } \\ \text { VPT } & \text { Vibration perception threshold }\end{array}$

\section{Introduction}

Diabetic somatic peripheral neuropathy (DPN) is a chronic and potentially disabling complication of diabetes, having a negative impact on physical and psychosocial functioning and on the quality of life of persons affected by this disorder [1-4]. DPN is not a single entity but encompasses a variety of clinical manifestations that can include unremitting pain, reduced feeling in the feet and postural instability. Moreover, DPN is a well-established major risk factor for foot ulceration and amputations [5, 6].

Evidence from individual studies [7-10], supported by a meta-analysis [11], suggests that there is a link between DPN and depressive symptoms. However, previous studies have focused almost exclusively on severe neuropathic pain, even though less than a third of patients with DPN experience painful symptoms [12] and many experience no symptoms [13]. Furthermore, the relationship between depression and other DPN symptoms (e.g. unsteadiness) has not been adequately addressed. As these reports are exclusively crosssectional in design, they cannot disentangle the temporal order of association. Generally, studies of depression and diabetes complications, including DPN, view them as comorbid conditions, each independently contributing to reduced mobility and physical dysfunction [14, 15], unemployment and work disability [16], and increased health service costs [17].

Several hypotheses have been proposed to explain the link between physical illness and depression. In keeping with the activity restriction model of depression [18], it is postulated that illness-related functional disability and restrictions in activities of daily living (ADL) are responsible for depressive affect. In the context of diabetes, for example, the results of a large community-based study indicate that functional limitations play an important role in the development of depression in people with type 2 diabetes [19]. This finding is of particular relevance to DPN in view of its association with severe physical disability, and in some patient populations it fully accounts for diabetes-related functional limitations [2]. Other researchers argue that the impact of ADL restrictions on depression may depend on the extent to which being unable to perform daily activities has a negative impact on sense of self [20]. Dyeson [21], for example, reported that the perception of the self as a burden on one's caregiver is a mediator of depressive symptoms among chronically ill care recipients. Furthermore, the self-regulatory model of illness [22] postulates that shared cognitions underlying physical illness and depressive affect create the possibility of a psychological path linking physical illness to depressive symptoms: both are perceived as chronic, uncontrollable and potentially having serious consequences.

Influenced by these theories, in a recent cross-sectional study we examined the relationship between DPN severity and depressive symptoms and explored the potential physical and psychosocial mediators of this association [23]. This study departed from the earlier reports in several important ways: (1) study participants were selected based on clinical tests of neurological dysfunction rather than the presence of neuropathic symptoms or foot ulceration and therefore were representative of a typical high-risk DPN population attending specialised diabetes clinics; and (2) rather than focusing on a single DPN symptom (e.g. pain) or foot ulceration alone, it assessed the independent contributions of a variety of DPN somatic experiences to depressive symptoms. The results of that study demonstrated that DPN is significantly associated with depressive symptoms, with neuropathy-related factors accounting for nearly half of the variance in depression scores. Neuropathic symptoms of pain, unsteadiness and reduced feeling in the feet each were independently associated with depressive symptoms and together accounted for the relationship between the clinical measures of neuropathy severity and depressive symptoms. Moreover, the association between neuropathic and depressive symptoms was partially mediated by two sets of relatively independent psychosocial factors: (1) illness cognitions, or perceptions of symptom unpredictability and the lack of treatment control; and (2) restrictions in ADL and diminished self-worth (self-perception as a family burden). However, the cross-sectional design of this report did not permit us to draw conclusions regarding the direction of the observed relationships.

The present follow-up study extends our cross-sectional model by examining the prospective relationships between neuropathy severity defined by clinical tests, foot ulceration, patient reports of neuropathic symptoms and 
neuropathy-related psychosocial consequences, and depressive symptoms over 18 months of follow-up. We hypothesised that both baseline and change in DPN-related physical and psychosocial factors would predict increments in depressive symptoms over time.

\section{Methods}

\section{Subjects}

A sample of 522 patients with diabetic peripheral neuropathy and either type 1 or type 2 diabetes was recruited at three sites: Manchester, UK; Baltimore, MD, USA; and State College, PA, USA. Permission to conduct this study was granted by the ethics committee at each site. Of the 522 patients, 495 agreed to participate and gave informed consent (participation rate 95\%). Detailed characteristics of the study population and methods used have been published previously [23]. Here we provide an overview of the study design.

As this was an investigation of patients at high risk of developing neuropathic foot ulcers, study participants were purposely selected to have moderate to severe neuropathy defined as vibration perception threshold (VPT) $>25 \mathrm{~V}$ and neuropathy disability score (NDS) $>3$; both these measures predict foot ulceration [24]. The VPT is a measure of large fibre dysfunction and the NDS is a composite score comprising both small (pain and temperature sensation) and large (vibration sensation and ankle reflexes) fibre dysfunction. Thus patients with mild neuropathic deficits were excluded from the study. Patients were also excluded if they had significant peripheral vascular disease, a history of major amputation, or other severe chronic medical diseases or complications of diabetes precluding participation.

Of the 495 participants who completed the baseline assessment, $376(76 \%)$ also completed the re-assessment at 9 months, and 338 (68\%) completed the final follow-up examination at 18 months. At the 18 month assessment, 44 participants were lost to follow-up because of death or poor health, 34 refused participation, 27 provided partial data, seven had moved away, and 45 were lost to follow-up for other or unknown reasons.

For this analysis we restricted the sample to the 338 participants with valid depressive symptoms data at the 18 month assessment. Of these participants, 313 also participated in the 9 month assessment. A meansubstitution approach was used to replace missing data for participants included in the main analyses.

\section{Measures}

Neuropathy Measures of neuropathy severity were raw scores for NDS and VPT. The presence of foot ulcers, defined as a full-thickness skin break below the malleoli, was determined by examination at baseline and 9 months. Past foot ulceration and the development of a new foot ulcer between the visits were obtained by asking each participant 'Have you (ever/since the last visit) had a foot ulcer (an open sore on your foot)?' supplemented by scrutiny of medical records.

Neuropathy symptoms were assessed by three scales from the Neuropathy and Foot Ulcer-specific Quality of Life Instrument (NeuroQoL), a neuropathy-specific quality of life instrument [4]: (1) neuropathic pain and/or paraesthesiae (Cronbach's $\alpha=0.87$ at baseline, 0.89 at 9 month follow-up); (2) symptoms of reduced feeling in the feet ( $\alpha=$ 0.90 at baseline, 0.91 at follow-up); and (3) unsteadiness ( $\alpha=0.97$ at baseline and follow-up).

DPN-related psychosocial consequences Neuropathy-related restrictions in ADL were assessed using a three-item NeuroQoL scale (alpha $=0.90$ at baseline, 0.93 at followup). Neuropathy-related social self-perception was measured by a three-item NeuroQoL scale: 'In the past 4 weeks how much have your foot problems interfered with your relationships with people close to you? Have you felt more physically dependent than you would like to be on people close to you? Has your role in the family changed as a result of your foot problems?' (alpha $=0.90$ at baseline, 0.91 at follow-up).

Depressive symptoms Consistent with our cross-sectional report [23], depressive symptoms were assessed with the seven-item subscale of the Hospital Anxiety and Depressive Scale (HADS), measuring depressive anhedonia (HADS-D) or the absence of both positive affect and pleasure from everyday tasks [25]. The HADS-D subscale (range 0-21) had an alpha of 0.82 at baseline and 0.83 at the 18 month follow-up.

Control variables Control variables included sex, age, education, marital status, type of diabetes, number of diabetes complications other than neuropathy, and number of comorbid disorders. Comorbid disorders included all self-reported medical conditions other than diabetes and its complications. Complications of diabetes, including retinopathy, nephropathy, and cardiovascular disease, were recorded by patients' self-report. Additionally, as there were significant differences between the UK and US participants [23], all analyses controlled for study site.

Change scores for predictors Measures of change for predictors (DPN symptoms, consequences) were created by subtracting the baseline value from the 9 month value. Thus, positive scores represent increases in the scores over time, negative scores represent decreases, and zero repre- 
sents no change. Controlling for the baseline values of predictors allowed assessment of the contributions of baseline and change in predictor values independently from each other.

\section{Statistical analysis}

All data were analysed using SPSS (Chicago, IL, USA). Values are shown as means \pm SD. Several different approaches were explored to address missing data in the main regression analyses. Although list-wise deletion of cases with any missing data was an option, it can lead to biased results [26] and would have reduced the study sample size to 274. Therefore we tested models using a variety of methods for imputing missing values for predictors (mean substitution, last observation carried forward, linear interpolation) as well as a model for all 495 cases using full information maximum likelihood estimation [27]. The results of these various approaches were essentially identical. Therefore, we are confident that the mean-substitution approach, results of which are presented below, is appropriate.

Analysis of sample attrition was performed by comparing the 338 participants who completed the 18 month follow-up with the 157 participants who did not complete the 18 month follow-up.

A progressive series of multivariate regression models was estimated to examine the hypothesised causal relations among the clinical measures of neuropathy severity, foot ulceration, patient reports of neuropathic symptoms and neuropathy-related psychosocial consequences, and depressive symptoms (results for each model are presented). Baseline neuropathy-specific measures were forced into the models; this was necessary to enable us to enter measures of change in these variables into subsequent models. Measures of change were forced into subsequent models. If neither the baseline nor change measure of a variable was significant, both were eliminated from the models; if either was significant, both were retained in the models to estimate their effects independently of each other. Thus, neuropathy-related variables shown in the results represent only those for which either baseline or change scores were significantly related to depressive symptoms.

Change in depressive symptoms was assessed by controlling for the baseline value of depressive symptoms. Controlling for baseline HADS-D allowed independent variables to be interpreted as predicting change in HADS$\mathrm{D}$ from baseline to 18 months, i.e. as accounting for the residual values after accounting for the variance shared with the baseline value. This strategy provided a measure of change in the predictors (until 9 months) that temporally precedes the outcome (change until 18 months). It also allowed an assessment of the stability of depressive symptoms over time (indicated by the association between baseline and 18 month depressive symptoms scores).

Procedures recommended by Baron and Kenny [28] were used to evaluate potential mediators. Sobel's variance estimate [29] was used as the test of whether the indirect effect of the independent variable on the dependent variable via the mediator was significantly different from zero.

\section{Results}

Characteristics of the study population are presented in Table 1: participants were patients with type 2 diabetes who were older and predominantly male. Participants were recruited to have moderate to severe neuropathy on clinical testing, and $14.7 \%$ had an active foot ulcer at baseline. Using the cut-off score of $>8$ on HADS-D, the percentage of likely cases of depression in our sample was $24.5 \%$ at baseline and $28.4 \%$ at follow-up; using the cut-off score of $>11$, the percentage of likely cases was $8.7 \%$ and $10.7 \%$, respectively.

There were significant differences at baseline between those who were included in the analytical sample and those who were lost to follow-up. Specifically, participants who were lost to follow-up $(n=157)$ had higher HADS-D scores $(6.06 \pm 4.58$ vs $4.77 \pm 3.70 ; p=0.002)$ and reported higher levels of neuropathic pain $(2.08 \pm 0.94$ vs $1.89 \pm 0.81$; $p=0.024)$.

No significant differences between baseline and followup values were observed in the analytical sample with the exception of depressive symptoms, which increased significantly over time. During the 9 month follow-up 14\% of the study participants developed new foot ulcers and $11 \%$ of patients had active foot ulcers at the 9 month follow-up visit.

Results of the main regression analyses are presented in Table 2. The full model explained $60 \%$ of the variance in HADS-D at 18 months, with $9 \%$ of the incremental variance accounted for by neuropathy-related physical and psychosocial factors.

Baseline predictors of increased depressive symptoms at 18 month follow-up

As expected, baseline HADS-D was highly associated with 18 month HADS-D $(\beta=0.68, p<0.001)$, indicating substantial stability in depressive symptoms over the 18 months of the study; none of the baseline demographic/disease variables was predictive of changes in HADS-D over time (see model I). Of the two baseline clinical measures of DPN severity only $\operatorname{NDS}(\beta=0.10, p=0.01)$ was significantly predictive of changes in HADS-D (see model II). When baseline DPN symptoms were added into the model, higher 
Table 1 Characteristics of the study population
Data are percentage or mean $\pm \mathrm{SD}$

${ }^{a}$ Significance tests assessed difference between participants in the analytical sample and those who did not complete follow-up

${ }^{\mathrm{b}}$ Follow-up at 9 month visit, except for HADS depressive symptoms which is the 18 month value; significance tests assessed the difference between baseline and follow-up values in the analytical sample: ${ }^{*} p<0.05$, $* * p<0.01$

${ }^{\mathrm{c}}$ Scores range from 1 to 5

NA, not applicable; SSRI, selective serotonin reuptake inhibitor

\begin{tabular}{|c|c|c|c|}
\hline Characteristic & $\begin{array}{l}\text { Baseline total sample } \\
(n=495)^{\mathbf{a}}\end{array}$ & $\begin{array}{l}\text { Baseline analytical sample } \\
(n=338)\end{array}$ & $\begin{array}{l}\text { Follow-up } \\
(n=338)^{\mathbf{b}}\end{array}$ \\
\hline Study site (UK) (\%) & 65.7 & 63.9 & NA \\
\hline Sex (male) $(\%)$ & 70.7 & 70.7 & NA \\
\hline Age (years) & $61.87 \pm 11.01$ & $61.24 \pm 10.81$ & NA \\
\hline \multicolumn{4}{|l|}{ Education $(\%)$} \\
\hline Primary & 3.1 & 3.0 & NA \\
\hline Secondary & 53.8 & 50.9 & NA \\
\hline Some college & 19.7 & 22.1 & NA \\
\hline College graduate & 14.1 & 14.8 & NA \\
\hline Postgraduate & 9.1 & 8.8 & NA \\
\hline Living alone $(\%)$ & 30.5 & 29.6 & NA \\
\hline Type 2 diabetes $(\%)$ & 73.0 & 73.0 & NA \\
\hline Number of diabetes complications & $1.56 \pm 1.10$ & $1.53 \pm 1.10$ & NA \\
\hline Number of concomitant disorders & $0.97 \pm 1.06$ & $0.99 \pm 1.08$ & NA \\
\hline Neuropathy disability score & $7.39 \pm 2.20$ & $7.42 \pm 2.23$ & NA \\
\hline Vibration perception threshold & $40.85 \pm 9.81$ & $41.56 \pm 9.55$ & \\
\hline Active foot ulcer & 15.9 & 14.7 & 11.3 \\
\hline \multicolumn{4}{|l|}{ NeuroQoL symptom scores } \\
\hline Pain $^{c}$ & $1.95 \pm 0.86^{*}$ & $1.91 \pm 0.83$ & $1.92 \pm 0.85$ \\
\hline Unsteadiness $^{\mathrm{c}}$ & $2.37 \pm 1.31$ & $2.35 \pm 1.28$ & $2.45 \pm 1.32$ \\
\hline Reduced feeling $^{\mathrm{c}}$ & $2.84 \pm 1.48$ & $2.88 \pm 1.49$ & $2.94 \pm 1.51$ \\
\hline NeuroQoL ADL restrictions ${ }^{c}$ & $2.51 \pm 1.38$ & $2.55 \pm 1.34$ & $2.51 \pm 1.38$ \\
\hline NeuroQoL social self-perception ${ }^{c}$ & $2.12 \pm 1.32$ & $2.10 \pm 1.26$ & $2.07 \pm 1.29$ \\
\hline HADS depressive symptoms & $5.19 \pm 4.05 * *$ & $4.93 \pm 3.79$ & $5.31 \pm 4.01 *$ \\
\hline \multicolumn{4}{|l|}{ Antidepressant medications (\%) } \\
\hline Tricyclics only & 11.0 & 13.3 & 12.4 \\
\hline SSRIs only & 5.7 & 6.5 & 6.8 \\
\hline Both & 1.0 & 1.2 & 0.9 \\
\hline
\end{tabular}

levels of unsteadiness at baseline significantly predicted increased depressive symptoms $(\beta=0.16, p=0.001)$ and the association of NDS with depressive symptoms was reduced to non-significance $(\beta=0.07, p=0.14$; see model III). Mediation analyses showed that unsteadiness was a significant mediator of the relationship between NDS and HADS-D changes $(z=2.75, p<0.01)$. Higher levels of restrictions in ADL at baseline (model IV) significantly predicted increased HADS-D $(\beta=0.16, p<0.01)$. Mediation analyses showed that restriction in ADL was a significant partial mediator of the relationship between unsteadiness and HADS-D changes $(z=2.69, p<0.01)$. Although not significant, baseline pain was retained in the model because change in pain was entered in subsequent models.

Change scores as predictors of increased depressive symptoms at 18 month follow-up

When change scores for pain and unsteadiness were added into the model, changes in pain $(\beta=0.09, p=0.02)$ and unsteadiness $(\beta=0.10, p=0.02)$ were each significant independent predictors of changes in HADS-D (see model V). Changes in ADL restrictions $(\beta=0.10, p=0.02)$ were entered in the next step and accounted for a significant increase in HADS-D (see model VI). Mediation analyses showed that changes in restrictions in ADL fell short of statistical significance as a mediator of the relationships of changes in HADS-D with changes in unsteadiness $(z=1.90$, $p<0.06)$ and changes in pain $(z=1.78, p<0.08)$. Changes in social self-perception significantly predicted changes in HADS-D ( $\beta=0.13, p=0.003$ ), reducing the relationship of changes in pain, unsteadiness and ADL restrictions with changes in depressive symptoms to non-significance (see model VII). Change in social self-perception was a significant mediator of the relationships of changes in depressive symptoms with changes in unsteadiness $(z=2.64, p<0.01)$ and changes in ADL restrictions $(z=2.72, p<0.01)$ but not changes in pain $(z=1.22, p=0.22)$.

To account for the potential confounding effects of antidepressants, the analyses reported above were replicated 
Table 2 Demographic, disease and psychosocial predictors of depressive symptoms in patients with diabetic peripheral neuropathy

\begin{tabular}{|c|c|c|c|c|c|c|c|}
\hline \multirow[t]{2}{*}{ Predictor } & \multicolumn{7}{|l|}{ Model } \\
\hline & I & II & III & IV & $\mathrm{V}$ & VI & VII \\
\hline Baseline depressive symptoms & $0.68 * * *$ & $0.67 * * *$ & $0.61 * * *$ & $0.56^{* * *}$ & $0.56^{* * *}$ & $0.56^{* * *}$ & $0.55 * * *$ \\
\hline \multicolumn{8}{|l|}{ Demographic/disease variables } \\
\hline Sex & 0.04 & 0.04 & 0.03 & 0.04 & 0.03 & 0.03 & 0.04 \\
\hline Education & -0.07 & -0.07 & -0.08 & -0.06 & -0.07 & -0.07 & -0.07 \\
\hline Type 2 diabetes & 0.05 & 0.06 & 0.05 & 0.05 & 0.04 & 0.04 & 0.06 \\
\hline Living with partner & -0.01 & -0.02 & -0.01 & -0.01 & 0.01 & -0.01 & -0.02 \\
\hline Age & -0.08 & -0.08 & -0.09 & -0.07 & -0.04 & -0.04 & -0.05 \\
\hline Study site (UK) & -0.01 & -0.01 & -0.02 & -0.05 & -0.04 & -0.04 & -0.04 \\
\hline Number of diabetes complications & 0.03 & 0.03 & 0.02 & 0.02 & 0.01 & 0.01 & 0.00 \\
\hline Number of co-morbidities & 0.01 & 0.02 & 0.01 & 0.01 & 0.01 & 0.01 & 0.02 \\
\hline \multicolumn{8}{|l|}{ Baseline DPN severity } \\
\hline NDS & & $0.10^{*}$ & 0.07 & 0.06 & 0.05 & 0.05 & 0.05 \\
\hline VPT & & -0.04 & -0.05 & -0.06 & -0.07 & -0.07 & -0.06 \\
\hline \multicolumn{8}{|l|}{ Baseline DPN symptoms } \\
\hline Pain & & & -0.02 & -0.04 & -0.02 & -0.04 & -0.04 \\
\hline Unsteadiness & & & $0.16^{*}$ & 0.10 & $0.15^{* *}$ & $0.13 *$ & $0.10^{*}$ \\
\hline Baseline ADL restrictions & & & & $0.16^{* *}$ & $0.14 * *$ & $0.21 * * *$ & $0.18^{* *}$ \\
\hline Baseline social-self perception & & & & 0.04 & 0.03 & 0.01 & 0.09 \\
\hline \multicolumn{8}{|l|}{ Change in DPN symptoms (0-9 months) } \\
\hline Pain & & & & & $0.09 *$ & $0.08^{*}$ & 0.07 \\
\hline Unsteadiness & & & & & $0.10^{*}$ & $0.08 *$ & 0.05 \\
\hline \multicolumn{8}{|l|}{ Change in $\mathrm{ADL}$ restrictions } \\
\hline (0-9 months) & & & & & & $0.10^{*}$ & 0.06 \\
\hline Change in social-self perception ( $0-9$ months $)$ & & & & & & & $0.13 * *$ \\
\hline$R^{2}$ change & $0.512 * * *$ & $0.009^{*}$ & $0.015 * * *$ & $0.023 * * *$ & $0.020 * *$ & $0.007 *$ & $0.012 * *$ \\
\hline
\end{tabular}

Data are standardised regression coefficients $(\beta)$

Each column represents a separate model; comparing coefficients across successive models indicates where variables added to a subsequent model mediate significant associations present in an earlier model

$* p<0.05 ; * * p<0.01 ; * * * p<0.001$

in two parallel analyses, one controlling for patients' use of antidepressant medication and the other excluding all participants reporting any antidepressant medication. These analyses produced essentially the same results as those presented above.

\section{Discussion}

The results of this longitudinal study are largely consistent with our earlier cross-sectional report that linked neuropathy to depressive symptoms through a series of DPN somatic experiences, resulting in ADL restrictions and diminished social self-perception [23]. In this longitudinal analysis we found that worse baseline neuropathy status was associated not only with more severe baseline depressive symptoms, but also with worsening depressive symptoms over time. Moreover, decline in neuropathyrelated physical and psychosocial functioning over time contributed to further increments in depressive symptoms. Specifically, a clinical measure of DPN severity (NDS) predicted an increase in depressive symptoms over time, and its effect was mediated by perceived symptoms and psychosocial consequences. In keeping with our crosssectional observations [23], unsteadiness was the symptom with the strongest association to depressive symptoms, and it was linked to depressive symptoms by the perceptions of diminished value of the self due to inability to perform family roles.

The observation that baseline levels of unsteadiness predict increments in depressive symptoms indicates that unsteadiness has a cumulative effect on depressive symptoms. That is, patients who experience unsteadiness at baseline are not only more likely to experience depressive symptoms at the time of 
the assessment, but are also likely to experience increased depressive symptoms in the future, independently of changes in unsteadiness over time. Worse yet from the patient perspective, increases in perceived unsteadiness over time further contribute to increases in depressive symptoms, irrespective of the patients' initial level of symptoms of unsteadiness. In contrast, only the change in pain severity (not baseline levels) was significantly related to change in depressive symptoms in our study, suggesting that the impact of pain is not cumulative over time.

The relationship between pain and depressive symptoms observed in our study is not unique. There is ample evidence of a cross-sectional association between chronic pain and depressive symptoms, but longitudinal studies have failed to yield consistent results [30,31]. One possible explanation for this lack of consistency is that longitudinal studies of the relationship of pain to depression may assess either absolute levels of depression at follow-up or change in depression over time. To see whether this explained why baseline pain was not related to depression in our main analyses, we re-ran our analyses predicting depressive symptoms at 18 months without controlling for baseline depressive symptoms; as we expected, the relationship of baseline pain to absolute depressive symptoms levels at follow-up was much larger and statistically significant. Thus, the reason that baseline pain was not associated with follow-up depressive symptoms in our main analyses was because there was an indirect relationship between baseline pain and follow-up depression; baseline pain was associated with baseline depressive symptom level, which in turn was associated with depressive symptoms at follow-up. By controlling for baseline depressive symptoms in our study, we removed the shared variance between baseline depression and follow-up depression from the model. Thus our analysis examined the ability of pain to predict follow-up depressive symptoms independently of its relationship to baseline depressive symptoms. In essence, baseline pain contributes to the chronicity of depressive symptoms while change in pain contributes to fluctuations of depressive symptoms over time.

We also explored the possibility that attrition bias might have accounted for the lack of the association between baseline levels of pain and increments in depressive symptoms. We conducted analyses that included participants who had dropped out by applying methods to estimate missing data (last observation carried forward and full information maximum likelihood) which take into account the higher levels of pain and depression among these participants; these analyses produced essentially the same results. Thus, it seems unlikely that attrition had a major influence on our results.

Our results provide some illumination of the potential mechanisms linking unsteadiness and depressive symptoms, but there may be additional pathways. Baseline levels of unsteadiness continue to be a significant predictor of increased depressive symptoms in the final model, suggesting that unsteadiness is related to depressive symptoms through mechanisms that are not fully accounted for by our model. It has been proposed, for example, that perceived unsteadiness probably measures not only balance dysfunction but also other domains of vulnerability, such as decreased physiological reserve and psychosocial factors including poor self-efficacy with walking and fear of falling [32]. These are established risk factors for functional decline [33] and might have provided additional linkages of unsteadiness to depressive symptoms.

Interestingly, although foot ulceration is not significantly associated with depressive symptoms in our main longitudinal model, in supplementary analyses that omitted measures of neuropathy severity we found that a positive history of foot ulceration obtained at baseline was significantly predictive of increased depressive symptoms over time. This relationship became non-significant, however, when measures of neuropathy severity entered the equation, thereby indicating that the relationship between past foot ulceration and increased depressive symptoms is probably spurious and that neuropathic symptoms are the most immediate mediator of the relationship between neuropathy and distress. Nonetheless, even though foot ulcers are not independently associated with depressive symptoms, they serve as a marker for an increased risk for elevated depressive symptoms and patients with foot ulcers should be carefully monitored to determine whether they are depressed, especially in the light of recent findings linking depressive disorders to increased mortality in patients presenting with their first foot ulcers [34].

Compared with earlier reports [10,35], in our sample there were fewer cases of moderate to severe depression. It is important to note, however, that in the earlier studies patients were selected based on severe neuropathic complications, i.e. they had diabetic foot syndrome [35] or unremitting neuropathic pain [10], and therefore are not comparable to our population, which was selected based on clinical tests of neuropathic deficits (NDS and VPT) and not the presence of severe neuropathic complications. Indeed, a substantial proportion of our patients were symptom-free. Thus, we believe that the results of our study are generalisable to a larger population, i.e. to patients with moderate to severe neuropathy. Indeed, in previous studies from our group we reported that $43 \%$ of a clinic-based diabetes cohort had VPT $>25$ [36], and $44 \%$ of a community cohort had NDS $>3$ [37], suggesting that these results apply to a substantial proportion of patients with diabetes.

Any attempt to project cause and effect between neuropathy and depression is fraught with difficulty. One strategy is to use a dose of tricyclic medication too low to achieve a physiological antidepressant effect, and observe whether there is a reduction in depression commensurate with the reduction in pain [38]. 
The longitudinal design used in this study can establish a necessary element of causal inference, i.e. temporal ordering. Moreover, the mediation analysis conducted in our study provides additional evidence for causal inference because it specifies the mechanisms through which neuropathy might influence depressive symptoms. Therefore, this study's analysis design permits the conclusion that the observed association between neuropathy and depressive symptoms is not due to depressive symptoms causing diabetes symptoms and functional disability, as suggested by other groups [39, 40]. It is important to note that our findings do not disprove that alternative hypothesis; addressing that question would require a longitudinal analysis which examines whether depressive symptoms at an earlier time point predict neuropathy levels, symptoms and consequences at a later time point (an analysis that was beyond the scope of this study). In fact, the actual causal relationship between depressive symptoms and neuropathy (and/or reports of neuropathic symptoms) is likely to be reciprocal. Resolution of this question awaits further research.

This study has important implications for the care of patients with DPN. Neuropathy-related physical and psychosocial dysfunction is a risk factor for depressive symptoms in patients with DPN, a point that should be a focus in clinical care. While neuropathic pain contributes to this relationship, unsteadiness and its psychosocial consequences play a major role in this relationship, a finding that may surprise many clinicians. Thus, clinicians should address unsteadiness as a key symptom when assessing patients with diabetic neuropathy and, pending a definitive intervention study, should consider a multifaceted approach to the management of these individuals. Such an approach would include a functional component (physical adaptation to instability to improve safety), a psychosocial component (strategies aimed at increasing participation in daily activities and enhanced perceptions of competence in important social roles), and perhaps a pharmacological component (the use of antidepressants). The importance of unsteadiness as a neuropathic symptom should receive attention in the education of both healthcare providers and patients.

Acknowledgements This work was supported by grants from Diabetes UK and the American Diabetes Association.

Duality of interest The authors declare that there is no duality of interest associated with this manuscript.

\section{References}

1. Boulton AJM, Malik RA, Arezzo J, Sosenko JM (2004) Diabetic neuropathy: technical review. Diabetes Care 27:1458-1487

2. Resnick HE, Stansberry KB, Harris TB et al (2002) Diabetes, peripheral neuropathy, and old age disability. Muscle Nerve 25:43-50
3. Resnick HE, Vinik AI, Schwartz AV et al (2000) Independent effects of peripheral nerve dysfunction on lower-extremity physical function in old age: the Women's Health and Aging Study. Diabetes Care 23:1642-1647

4. Vileikyte L, Peyrot M, Bundy CE et al (2003) The development and validation of a neuropathic symptom and foot ulcer-specific quality of life instrument. Diabetes Care 26:2549-2555

5. Reiber GE, Vileikyte L, Boyko EJ et al (1999) Causal pathways for incident lower-extremity ulcers in patients with diabetes from two settings. Diabetes Care 22:157-162

6. Pecoraro R, Reiber GE, Burgess EM (1990) Pathways to diabetic limb amputation. Diabetes Care 13:513-521

7. Winocour PH, Main CJ, Medlicott G, Anderson DC (1990) A psychometric evaluation of adult patients with type I (insulindependent) diabetes mellitus: prevalence of psychological dysfunction and relationship to demographic variables, metabolic control and complications. Diabetes Res 14:171-176

8. Viinamäki H, Niskanen L, Uusitupa M (1995) Mental well-being in people with non-insulin-dependent diabetes. Acta Psychiatr Scand 92:392-397

9. Geringer ES, Perlmuter LC, Stern TA, Nathan DM (1988) Depressive symptoms and diabetic neuropathy: a complex relationship. J Geriatr Psychiatry Neurol 1:11-15

10. Gore M, Brandenburg NA, Dukes E, Hoffman DL, Tai KS, Stacey B (2005) Pain severity in diabetic peripheral neuropathy is associated with patient functioning, symptom levels of anxiety and depressive symptoms, and sleep. J Pain Symptom Manage 30:374-385

11. de Groot M, Anderson R, Freedland KE, Clouse RE, Lustman PJ (2001) Association of depressive symptoms and diabetes complications: a meta-analysis. Psychosom Med 63:619-630

12. Davies M, Brophy S, Williams R, Taylor A (2006) The prevalence, severity, and impact of painful diabetic peripheral neuropathy in type 2 diabetes. Diabetes Care 29:1518-1522

13. Dyck PJ, Kratz KM, Karnes JL et al (1993) The prevalence by staged severity of various types of diabetic neuropathy, retinopathy, and nephropathy in a population-based cohort: the Rochester Diabetic Neuropathy Study. Neurology 43:817-824

14. Bruce DG, Davis WA, Davis TM (2005) Longitudinal predictors of reduced mobility and physical disability in patients with type 2 diabetes: the Fremantle Diabetes Study. Diabetes Care 28:24412447

15. Von Korff M, Katon W, Lin EH et al (2005) Potentially modifiable factors associated with disability among people with diabetes. Psychosom Med 67:233-240

16. Von Korff M, Katon W, Lin EH et al (2005) Work disability among individuals with diabetes. Diabetes Care 28:1326-1332

17. Simon GE, Katon WJ, Lin EH et al (2005) Diabetes complications and depressive symptoms as predictors of health service costs. Gen Hosp Psych 27:344-351

18. Williamson GM (1998) The central role of restricted normal activities in adjustment to illness and disability: a model of depressed affect. Rehab Psychol 43:327-347

19. Pouwer F, Beekman AT, Nijpels G et al (2003) Rates and risks for co-morbid depressive symptoms in patients with type 2 diabetes mellitus: results from a community-based study. Diabetologia 46:892-898

20. Heidrich SM, Forsthoff CA, Ward SE (1994) Psychological adjustment in adults with cancer: the self as mediator. Health Psychol 13:346-353

21. Dyeson TB (2000) Burden self-image: a mediating variable of depressive symptoms among chronically ill care recipients. J Gerontol Soc Work 33:17-33

22. Leventhal H, Meyer D, Nerenz D (1980) The common sense representation of illness danger. In: Rachman $\mathrm{S}$ (ed) Contributions to medical psychology, vol 2. Pergamon, New York, pp 7-30 
23. Vileikyte L, Leventhal H, Gonzalez JS et al (2005) Diabetic peripheral neuropathy and depressive symptoms: the association revisited. Diabetes Care 28:2378-2383

24. Young MJ, Boulton AJM, Macleod AF, Williams DRR, Sonksen PH (1993) A multicentre study of the prevalence of diabetic peripheral neuropathy in the United Kingdom hospital clinic population. Diabetologia 36:150-154

25. Zigmond AS, Snaith RP (1983) The hospital anxiety and depressive symptoms rating scale. Acta Psychiatr Scand 67: 361-370

26. Schafer JL, Graham JW (2002) Missing data: our view of the state of the art. Psychol Methods 7:147-177

27. Muthén LK, Muthén BO (1998) Mplus user's guide. Muthen \& Muthen, Los Angeles

28. Baron RM, Kenny DA (1986) The moderator/mediator variable distinction in social psychological research: conceptual, strategic, and statistical considerations. J Pers Soc Psychol 51:11731182

29. Sobel ME (1982) Asymptotic intervals for indirect effects in structural equation models. In: Leinhart $\mathrm{S}$ (ed) Sociological methodology. Jossey Bass, San Francisco, pp 290-312

30. Smedstad LM, Vaglum P, Moum T, Kvien TK (1997) The relationship between psychological distress and traditional clinical variables: a 2 year prospective study of 216 patients with early rheumatoid arthritis. Br J Rheumatol 36:1304-1311

31. Dickens C, McGowan L, Clark-Carter D, Creed F (2002) Depressive symptoms in rheumatoid arthritis: a systematic review of the literature with meta-analysis. Psychosom Med $64: 52-60$
32. Lindenberger EC, Landefeld CS, Sands LP et al (2003) Unsteadiness reported by older hospitalized patients predicts functional decline. J Am Geriatr Soc 51:621-626

33. McKee KJ, Orbell S, Austin CA et al (2002) Fear of falling, falls efficacy, and health outcomes in older people following hip fracture. Disabil Rehabil 24:327-333

34. Ismail K, Winkley K, Stahl D et al (2007) A cohort study of people with diabetes and their first foot ulcer: the role of depressive symptoms on mortality. Diabetes Care 30:1473-1479

35. Simson U, Nawarotzky U, Porck W et al (2008) Depression, anxiety, quality of life and type D pattern among inpatients suffering from diabetic foot syndrome. Psychother Psychosom Med Psychol 58:44-50

36. Abbott CA, Carrington AL, Ashe H et al (2002) The North-West Diabetes Foot Care Study: incidence of, and risk factors for, new diabetic foot ulceration in a community-based patient cohort. Diabetic Med 19:377-384

37. Abbott CA, Garrow AP, Carrington AL et al (2005) Foot ulcer risk is lower in South-Asian and African-Caribbean compared with European diabetic patients in the U.K.: the North-West diabetes foot care study. Diabetes Care 28:1869-1875

38. Max MB, Culnane M, Schafer SC et al (1987) Amitriptyline relieves diabetic neuropathy pain in patients with normal or depressed mood. Neurology 37:589-596

39. Ciechanowski PS, Katon WJ, Russo JE, Hirsch IB (2003) The relationship of depressive symptoms to symptom reporting, selfcare and glucose control in diabetes. Gen Hosp Psych 25:246-252

40. Ludman EA, Katon W, Russo J et al (2004) Depressive symptoms and diabetes symptom burden. Gen Hosp Psych 26:430-436 